\title{
Large stimulated Brillouin scattering amplification in gases
}

\section{Fan Yang, Flavien Gyger, Luc Thévenaz}

Fan Yang, Flavien Gyger, Luc Thévenaz, "Large stimulated Brillouin scattering amplification in gases," Proc. SPIE 11199, Seventh European Workshop on Optical Fibre Sensors, 1119933 (28 August 2019); doi: 10.1117/12.2540660

Event: Seventh European Workshop on Optical Fibre Sensors, 2019, Limassol, Cyprus 


\title{
Large stimulated Brillouin scattering amplification in gases
}

\author{
Fan Yang, Flavien Gyger and Luc Thévenaz \\ EPFL Swiss Federal Institute of Technology, Group for Fibre Optics (GFO), SCI-STI-LT \\ Station 11, CH-1015 Lausanne, Switzerland
}

\begin{abstract}
We exploit backward stimulated Brillouin scattering in gases to achieve unprecedented nonlinear optical amplification in fibers. The gain coefficient is 10 times larger than any reported nonlinear gain in gas-filled HC-PCF and 6 times larger than the largest nonlinear gain in standard silica single-mode fiber (SMF). Furthermore, our system can work at any wavelength from vacuum ultraviolet to mid-infrared thanks to the nature of stimulated Brillouin scattering. This massive gain enables us to realize a single-pass gas Brillouin laser with $140 \mathrm{~mW}$ threshold power over $50 \mathrm{~m}$ of fibre. This platform opens new avenues in gas Brillouin lasing, all-optical signal processing and high performance distributed fiber sensing.
\end{abstract}

\section{INTRODUCTION}

Gas-filled hollow-core photonic crystal fibers (HC-PCFs), with small mode area, low propagation loss and high optical damage threshold have led to many innovative results, ${ }^{1}$ including soliton pulse compression, ${ }^{2,3}$ electromagnetically induced transparency, ${ }^{4}$ gas stimulated Raman scattering ${ }^{5,6}$ and gas forward stimulated Brillouin scattering. ${ }^{7}$ The peak forward Raman gain coefficients in hydrogen saturate due to collision broadening to values of $1.5 \mathrm{~cm} / \mathrm{GW}$ for vibrational transition and $0.5 \mathrm{~cm} / \mathrm{GW}$ for rotational transition at pressures above 3 bar. ${ }^{1}$ The peak backward Raman gain coefficient is much smaller than in the forward case (because of strong Doppler broadening) and saturates at higher pressure $(\sim 30$ bar $)$. The peak forward Brillouin gain coefficient is $9 \times 10^{-4} \mathrm{~m}^{-1} \mathrm{~W}^{-1}$ for atmospheric gas-filled HC-PCF, which is $\sim 300$ times smaller than the peak backward Brillouin gain coefficient in standard single-mode fiber (SMF). The forward SBS gain is proportional to the gas pressure, ${ }^{7,8}$ so by increasing the gas pressure, the gain can be enhanced but remains limited.

Here, we demonstrate that stimulated backward Brillouin scattering (SBS) can be substantially enhanced in gases and shows attractive properties, illustrated by the realisation of a gas Brillouin laser in a gas-filled HC-PCF. The large gain is due to the quadratic gas pressure dependence ${ }^{9}$ and the tight confinement of light over long distances, which means that although the gain is small at atmospheric pressure, it can be greatly enhanced by increasing pressure. We measured the backward SBS gain in 1 bar $\mathrm{CO}_{2}$-filled $\mathrm{HC}$-PCF to be $1 \times 10^{-3} \mathrm{~m}^{-1} \mathrm{~W}^{-1}$ which is $\sim 240$ times smaller than in standard SMF. However, by increasing the pressure to $\sim 40$ bar, we demonstrate a gain 10 times larger than any reported nonlinear gain in gas-filled HC-PCF and 6 times larger than the gain in silica-core SMF. This has enabled us to realize and demonstrate a gas Brillouin laser with $140 \mathrm{~mW}$ threshold power over a $50 \mathrm{~m} \mathrm{HC-PCF} \mathrm{(} \sim 1 \mathrm{~W}$ over the same SMF length). Compared with past free-space experiments (short interaction length and large beam size) in which megawatt pump pulsed laser were needed to observe SBS in gas, ${ }^{10-12}$ the threshold has been drastically decreased by several orders of magnitude. Furthermore, a novel strain-insensitive distributed temperature sensor can thus be considered with this platform because the fluid nature of gases makes it by essence insensitive to strain.

\section{BACKWARD SBS IN GAS-FILLED HC-PCF}

Pump and probe waves counter-propagate along a $\mathrm{HC}-\mathrm{PCF}$ and their beat note produces a longitudinally moving fringe pattern (Fig. 1(a)). When the phase matching condition is met, electrostriction gives rise to a travelling compressing wave in the gas, causing a Bragg-type coupling between pump and probe. Here, the frequency of the pump light is higher than the probe's frequency so that the probe is amplified by the pump if the frequency difference matches the Brillouin frequency shift $\nu_{\mathrm{B}}$ (i.e. the frequency for perfect phase matching), in total similarity with the process in solid-core optical fibres. The peak SBS gain is given by: ${ }^{9}$

Correspondence: Fan Yang, e-mail: fan.yang@epfl.ch

Seventh European Workshop on Optical Fibre Sensors, edited by Kyriacos Kalli, Gilberto Brambilla, Sinead O'Keeffe Proc. of SPIE Vol. 11199, 1119933 - (C) 2019 SPIE · CCC code: 0277-786X/19/\$21 · doi: 10.1117/12.2540660 
(a)
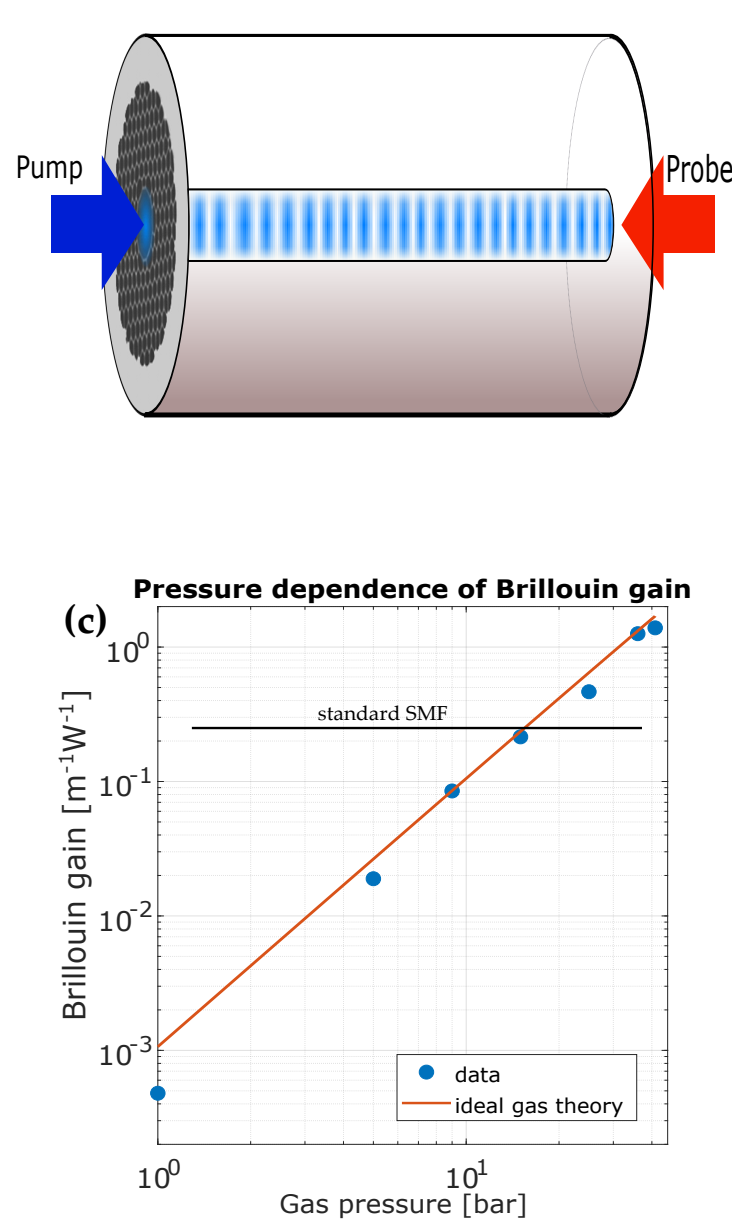

(b)

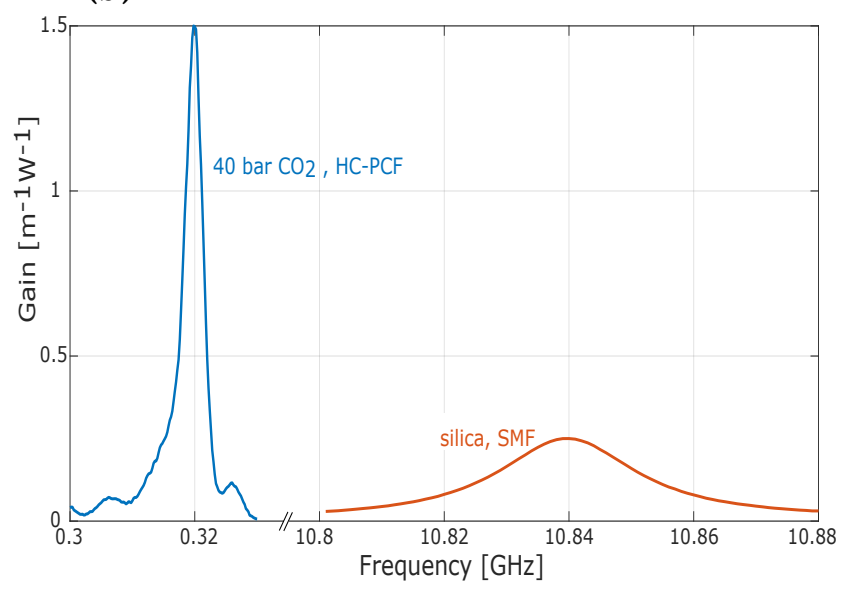

Pressure dependence of Brillouin linewidth

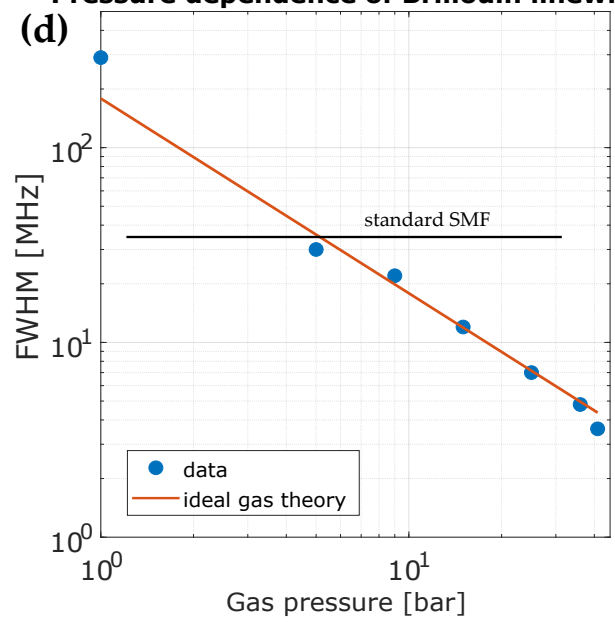

Figure 1. Backward SBS gain. (a) Schematic setup for backward SBS gain measurement. (b) Gain spectra for the HC-PCF filled with 40 bar $\mathrm{CO}_{2}$ and for a standard silica single-mode fiber (SMF). Horizontal axis is broken. (c) Peak Brillouin gain and (d) linewidth (FWHM) for $\mathrm{CO}_{2}$-filled $\mathrm{HC}-\mathrm{PCF}$ at different pressures. The black lines in (c) and (d) are the gain and linewidth of SBS in SMF, respectively.

$$
g_{0}=\frac{\gamma_{\mathrm{e}}^{2} \omega^{2}}{\rho n v c^{3} \Gamma_{\mathrm{B}} \mathrm{A}_{\mathrm{eff}}}
$$

where $\gamma_{\mathrm{e}}$ is the electrostrictive coefficient, $\omega$ is the light angular frequency, $\rho$ is the gas density, $n$ is the gas refractive index, $v$ is the acoustic velocity, $c$ is the light velocity in vacuum, $\Gamma_{\mathrm{B}} / 2 \pi$ is the gain spectrum linewidth (FWHM) and $\mathrm{A}_{\text {eff }}$ is the effective optical mode area of the fiber.

Within the ideal gas model, the linewidth and the electrostrictive coefficient can be expressed as: ${ }^{9}$

$$
\begin{aligned}
\Gamma_{\mathrm{B}} & =\frac{4}{3} \frac{\eta_{\mathrm{s}} q^{2}}{\rho} \\
\gamma_{\mathrm{e}} & =\rho \frac{\partial \epsilon}{\partial \rho}
\end{aligned}
$$

where $\eta_{\mathrm{s}}$ is the shear viscosity coefficient and $q$ is the acoustic wavevector. 
Since $\eta_{\mathrm{s}}$ and $\frac{\partial \epsilon}{\partial \rho}$ are independent of the gas pressure, the Brillouin linewidth is inversly proportional to the pressure while the electrostrictive coefficient is proportional to this quantity. As a result, these 2 trends identically contribute to make the gain grow with pressure, eventually exhibiting a square dependence on the gas pressure.

To minimize the impact of the residual pump reflection, both pump and probe are intensity modulated at distinct frequencies, so that only the nonlinear response proportional to the product of the pump and probe intensities is detected at the difference of the frequencies. The tested HC-PCF has a core diameter of $10 \mu \mathrm{m}$ and a loss of $16 \mathrm{~dB} / \mathrm{km}$. Each end of the $50 \mathrm{~m} \mathrm{HC}-\mathrm{PCF}$ is inserted into a ferrule and butt coupled to an angled cleaved SMF pigtail to minimize the pump reflection. The gas is pressurized inside the HC-PCF through the tiny gap between HC-PCF and SMF. Figure 1(b) shows the measured backward SBS gain spectrum in the HC-PCF filled with 40 bar carbon dioxide and, for comparison purpose, in a standard silica core SMF. It can be seen that the Brillouin frequency is in the sub-GHz range in the gas and much narrower. The side peaks are probably due to the presence of higher order optical and acoustic modes. Figures 1(c) and 1(d) show that the measured and theoretical peak gains and linewidths match very well. The gain is proportional to the square of the pressure while the linewidth is inversely proportional to the pressure, which are both in agreement with the theoretical model yielding Eq. (1) and Eq. (2). ${ }^{9}$

The measured backward SBS gain for different gases such as $\mathrm{N}_{2}, \mathrm{CH}_{4}$, and $\mathrm{CO}_{2}$ under the same condition (at 10 bar pressure and $24{ }^{\circ} \mathrm{C}$ ) are shown in Table 1. The gain of $\mathrm{CO}_{2}$ is 3 times larger than $\mathrm{CH}_{4}$ and 4 times larger than $\mathrm{N}_{2}$. The Brillouin frequency shift is different for these three types of gas which means the Brillouin frequency shift can be tuned by using different type of gases, or even a mixture for continuous tuning.

Table 1. Backward SBS in gas-filled $\mathrm{HC}-\mathrm{PCF}$ with 10 bar $\mathrm{N}_{2}, \mathrm{CH}_{4}$ and $\mathrm{CO}_{2}$ at $24{ }^{\circ} \mathrm{C}$.

\begin{tabular}{llrrr} 
Gas & Name & $\nu_{\mathrm{B}}[\mathrm{MHz}]$ & $\Gamma_{\mathrm{B}} / 2 \pi[\mathrm{MHz}]$ & $g_{0}\left[\mathrm{~m}^{-1} \mathrm{~W}^{-1}\right]$ \\
\hline $\mathrm{N}_{2}$ & Nitrogen & 451 & 41 & 0.025 \\
$\mathrm{CH}_{4}$ & Methane & 580 & 40 & 0.034 \\
$\mathrm{CO}_{2}$ & Carbon dioxide & 351 & 21 & 0.105
\end{tabular}

\section{SINGLE-PASS GAS BRILLOUIN LASER}
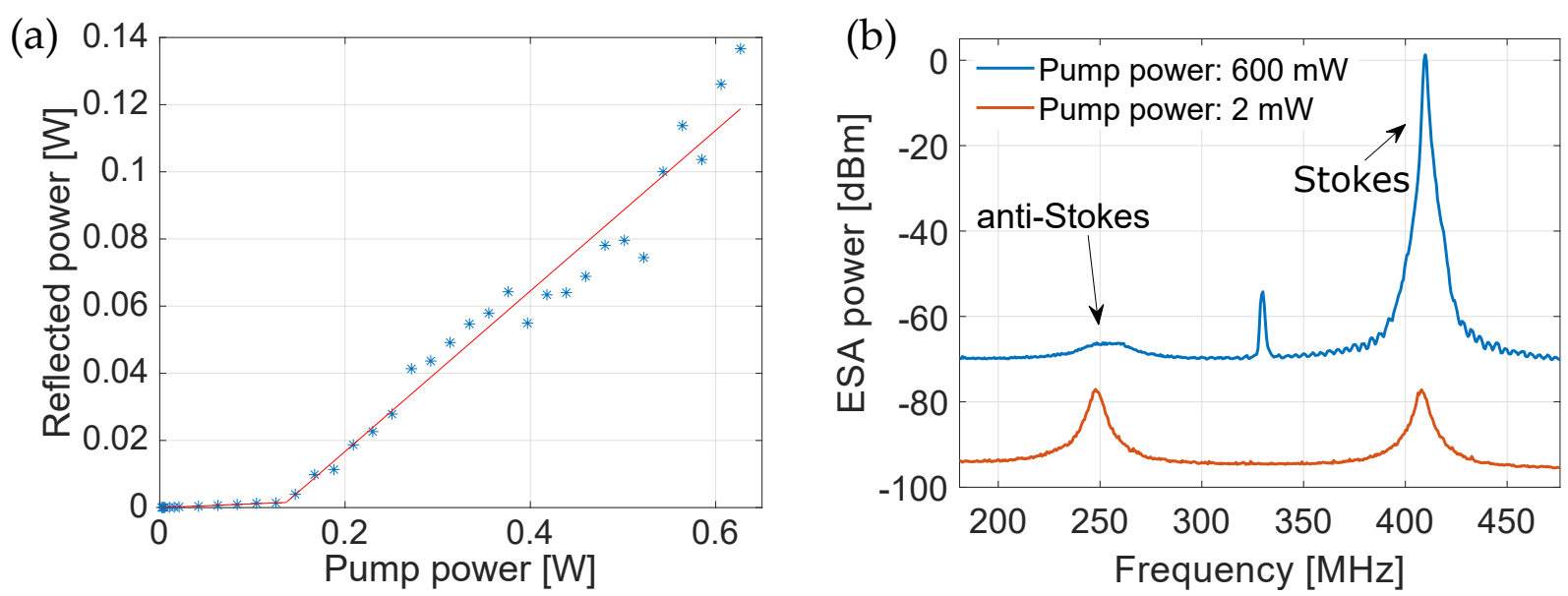

Figure 2. Single-pass gas Brillouin laser. (a) Reflected Stokes power as a function of input pump power into the HCPCF. (b) Reflected Stokes and anti-Stokes spectra with pump power of 2 and $600 \mathrm{~mW}$ are measured using an electrical spectrum analyzer (ESA) after heterodyning with the original laser light. This latter is frequency-shifted by an $80 \mathrm{MHz}$ acousto-optical modulator (AOM) to discriminate Stokes and anti-Stokes components.

In this experiment, backward SBS in the gas-filled HC-PCF is measured using CW pumping and a heterodyne detection after mixing with the pump light, upshifted in frequency by $80 \mathrm{MHz}$. The critical power (so-called "threshold") for SBS in 38 bar $\mathrm{CO}_{2}$-filled $\mathrm{HC}-\mathrm{PCF}$ is observed to be $140 \mathrm{~mW}$ for a $50 \mathrm{~m}$ long fibre, obtained by measuring the Stokes output power as a function of the input pump power (Fig. 2(a)). Figure 2(b) shows 
the backscattered spectra measured for 2 pump powers using an electrical spectrum analyzer. When the pump power is below threshold (i.e., $2 \mathrm{~mW}$ ), both Stokes and anti-Stokes show similar amplitudes. When the pump power is above threshold (i.e., $600 \mathrm{~mW}$ ), the Stokes component turns much higher than the anti-Stokes, which is a clear signature of a highly stimulated process. We also clearly observe the Stokes linewidth narrowing for increasing pump powers, in full agreement with the theory. ${ }^{13}$

\section{DISCUSSION AND CONCLUSION}

This SBS gain can be still much enhanced by further increasing the pressure inside the HC-PCF. Such fibers can easily sustain a pressure in the kilobar range by virtue of their small core diameter and thick glass sheath. ${ }^{1}$ The theoretical gain for 1,000 bar $\mathrm{CO}_{2}$ is anticipated to be more than 400 times larger than the gain achieved in this preliminary work using a gas pressure of $\sim 40$ bar. Using a similar platform, we can also investigate the light-sound interaction in supercritical fluid in the HC-PCF. It should be pointed out that the gas consumption in volume for a $50 \mathrm{~m}$ long $\mathrm{HC}-\mathrm{PCF}$ filled with 1,000 bar gas is equivalent to only $4 \mathrm{ml}$ at atmospheric pressure, thanks to the microscopic core size.

By changing the gas pressure, we evidenced that we can scale the gain, but moreover modify the linewidth and hence the acoustic lifetime (acoustic lifetime is proportional to the gas pressure within the ideal gas approximation), which is very distinctive of light-sound interactions in solid materials. Manipulating the acoustic lifetime turns out to be a very important feature for building Brillouin photon or phonon lasers. ${ }^{14}$

The use of pressurized gases is very attractive owing to their high transparency throughout the ultraviolet, visible and infrared regions. Nowadays, HC-PCFs showing broad transmission windows from ultraviolet to mid-infrared are available. ${ }^{15,16}$ Unlike the typical gas lasers requiring a pump frequency locked on a resonant transition between energy levels and emitting at fixed wavelengths using distinct transitions, our Brillouin laser can flexibly operate at any wavelength within the broad transmission band of the gas-filled HC-PCF.

Furthermore, the gas-filled HC-PCF can be sealed with trapped gas by splicing both ends to standard SMFs to make a perfectly airtight compact all-fiber gas cell with no bulk optics.

In summary, we have reported, for the first time, a huge nonlinear gain in gas and a single-pass gas Brillouin laser based on gas-filled HC-PCF with $140 \mathrm{~mW}$ power threshold. We have designed a general platform for lightsound interaction in gas, with possibilities of large amplification and sophisiticated all-optical signal processing ${ }^{17}$ in hollow-core fibers.

\section{Acknowledgement}

We acknowledge support from the Swiss National Foundation under grant agreement No. 178895 and 159897.

\section{REFERENCES}

1. P. St. J. Russell, et al., Nature Photonics 8, 278-286 (2014).

2. D. G. Ouzounov, et al., Science 301, 1702-1704 (2003).

3. N. Y. Joly, et al., Physical Review Letters 106, 203901 (2011).

4. S. Ghosh, J. E. Sharping, D. G. Ouzounov, and A. L. Gaeta, Physical Review Letters 94, 093902 (2005).

5. F. Benabid, J. C. Knight, G. Antonopoulos, and P. St. J. Russell, Science 298, 399-402 (2002).

6. F. Couny, F. Benabid, and P. S. Light, Physical Review Letters 99, 143903 (2007).

7. W. H. Renninger, R. O. Behunin, P. T. Rakich, Optica 3, 1316-1319 (2016).

8. Y. Zhao, et al., in 26th International Conference on Optical Fiber Sensors, (2018), paper WE1.

9. R. W. Boyd, Nonlinear Optics (Academic Press, 2008).

10. E. E. Hagenlocker and W. G. Rado, Applied Physics Letters 7, 236-238 (1965).

11. T. J. Greytak and G. B. Benedek, Physical Review Letters 17, 179-182 (1966).

12. C. Y. She, G. C. Herring, H. Moosmuller, and S. A. Lee, Physical Review Letters 51, 1648-1651 (1983).

13. A. L. Gaeta and R. W. Boyd, Physical Review A 44, 3205-3209 (1991).

14. N. T. Otterstrom, R. O. Behunin, E. A. Kittlaus, Z. Wang, and P.T. Rakich, Science 360, 1113-1116 (2018).

15. F. Kottig, et al., Nature Communications 8, 813 (2017).

16. J. C. Knight, in Optical Fiber Communication Conference, (Optical Society of America, 2019), paper Th3E.4.

17. M. Santagiustina, et al., Scientific Reports 3, 1594 (2013). 